University of Nebraska - Lincoln

DigitalCommons@University of Nebraska - Lincoln

\title{
Cross-Layer Packet Size Optimization for Wireless Terrestrial, Underwater, and Underground Sensor Networks
}

Mehmet C. Vuran

University of Nebraska-Lincoln, mcvuran@cse.unl.edu

Ian F. Akyildiz

Georgia Institute of Technology, Atlanta, GA, ian@ece.gatech.edu

Follow this and additional works at: https://digitalcommons.unl.edu/cseconfwork

Part of the Computer Sciences Commons

Vuran, Mehmet C. and Akyildiz, Ian F., "Cross-Layer Packet Size Optimization for Wireless Terrestrial, Underwater, and Underground Sensor Networks" (2008). CSE Conference and Workshop Papers. 145. https://digitalcommons.unl.edu/cseconfwork/145

This Article is brought to you for free and open access by the Computer Science and Engineering, Department of at DigitalCommons@University of Nebraska - Lincoln. It has been accepted for inclusion in CSE Conference and Workshop Papers by an authorized administrator of DigitalCommons@University of Nebraska - Lincoln. 


\section{Cross-layer Packet Size Optimization for Wireless Terrestrial, Underwater, and Underground Sensor Networks}

\author{
Mehmet C. Vuran \\ Department of Computer Science and Engineering \\ University of Nebraska-Lincoln, Lincoln, NE 68588 \\ Tel: (402) 472-5019 Fax: (402) 472-7767 \\ Email: mcvuran@cse.unl.edu
}

\author{
Ian F. Akyildiz \\ Broadband Wireless Networking Laboratory \\ School of Electrical \& Computer Engineering \\ Georgia Institute of Technology, Atlanta, GA 30332 \\ Tel: (404) 894-5141 Fax: (404) 894-7883 \\ Email: ian@ece.gatech.edu
}

\begin{abstract}
In this paper, a cross-layer solution for packet size optimization in wireless sensor networks (WSN) is introduced such that the effects of multi-hop routing, the broadcast nature of the physical wireless channel, and the effects of error control techniques are captured. A key result of this paper is that contrary to the conventional wireless networks, in wireless sensor networks, longer packets reduce the collision probability. Consequently, an optimization solution is formalized by using three different objective functions, i.e., packet throughput, energy consumption, and resource utilization. Furthermore, the effects of end-to-end latency and reliability constraints are investigated that may be required by a particular application. As a result, a generic, cross-layer optimization framework is developed to determine the optimal packet size in WSN. This framework is further extended to determine the optimal packet size in underwater and underground sensor networks. From this framework, the optimal packet sizes under various network parameters are determined.
\end{abstract}

\section{INTRODUCTION}

The unique characteristics of Wireless Sensor Networks (WSN) necessitate rethinking of classical wireless networking in all aspects [1]. Among these, the determination of the optimal packet size constitutes a fundamental and still an unexplored problem in WSN. Especially, the cross-layer effects of communication due to low power communication constraints and intrinsic properties of low cost sensor nodes call for a cross-layer assessment of optimal packet size for communication in WSN.

Traditionally, packet size optimization is performed considering a point-to-point link, where the goal is to ensure a successful and efficient transmission based on an efficiency metric [12], [14], [15]. However, in this traditional approach, the influence of multi-hop and broadcast nature of wireless communication in WSN cannot be captured. It is well-known that the packet size directly affects the reliability of the communication since longer packet sizes are susceptible to wireless channel errors given a certain level of link quality. However, in multi-hop WSN, the quality of the communication links depend on the routes established in the network. Moreover, the existence of neighbor nodes that contend for the shared wireless medium affect the communication performance significantly leading to degradation in communication success. Furthermore, the communication success also depends on both the characteristics of the wireless channel and the error control technique deployed. Considering these various factors that originate from different layers of the communication stack, a cross-layer assessment of packet size optimization for WSN is crucial.
Another aspect in packet size optimization is the nature of the WSN. WSN has found wide application areas since the development of highly sophisticated wireless sensor nodes [1]. These networks are mainly being deployed in terrestrial areas such as forests, factories, buildings, etc. In addition, recently, WSN research has developed in to underwater and underground environments. Underwater acoustic wireless sensor networks (UW-ASN) are characterized by very low bandwidth and high error rate underwater channels [2]. Similarly, recently, the applications and requirements of wireless underground sensor networks (WUSN) have been investigated [4], [13]. These networks impose additional challenges in terms of channel characteristics. As a result, an optimal packet size for these environments should also be determined.

In this paper, a cross-layer solution for packet size optimization in wireless terrestrial, underwater, and underground sensor networks is presented such that the cross-layer effects of multi-hop routing, the broadcast nature of the wireless channel, and the effects of error control techniques are captured. For terrestrial sensor networks, the effect of packet length on the collision probability is investigated. Moreover, the relationship between routing decisions and the packet size is highlighted. Furthermore, the effects of packet size on different performance metrics such as throughput, energy consumption, latency, and success rate are investigated considering these cross-layer effects. Finally, requirements of various types of applications in WSN are considered to develop a comprehensive framework for packet size optimization. The results of this framework provide optimal packet size values tailored for different application types. The optimization framework is also extended to determine the optimal packet size in two challenged environments, where wireless sensor networks find application areas. More specifically, underwater and underground environments are investigated.

The remainder of the paper is organized as follows: In Section II, related work on packet size optimization in wireless networks is summarized. The effects of packet size on collisions are investigated in Section III. Based on this investigation, in Section IV, the cross-layer solution for packet size optimization is presented. The results of the optimization framework are presented in Section V. We further extend our work for underwater and underground environment in Section VI. Finally, the paper is concluded in Section VII. 


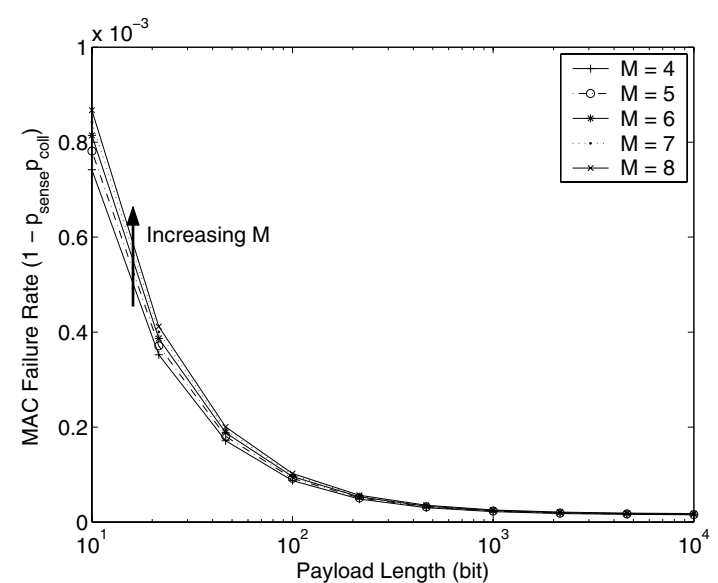

Fig. 1. MAC failure rate vs. payload length for different values of $M$.

\section{RELATED WORK}

Packet size optimization is a highly investigated topic in cellular networks [14]. However, the existing work mainly considers a single hop communication and performs optimization accordingly. Therefore, these results cannot be directly applied to WSN because of the multi-hop paradigm.

In [12], adaptive frame sizes are determined based on the varying properties of the wireless channel, i.e., for bad channel conditions, shorter packets with powerful error correction is used while longer packet sizes are selected for good channel conditions by which a high reliability can be achieved. However, variable packet sizes are not preferred in WSN due to strict hardware and computation constraints of wireless sensor nodes. As a result, we advocate to use fixed packet sizes.

The most relevant work on this topic is [15], where an optimal packet size framework is proposed. In this work, the effects of error correction on energy efficiency are investigated to determine the optimal packet size based on an energy channel model. However, the energy channel model is based on single hop behavior and the effects of multi-hop routing or MAC collisions are not addressed in [15].

\section{Factors Affecting The Packet Size}

In this section, we highlight the factors that affect the optimal packet size in sensor networks with a special focus on energy consumption. The energy consumption in WSN mainly depends on the energy required to transmit a packet and the reliability of the network. Usually small packet sizes lead to increased reliability due the decreased chance of bit errors over the wireless channel. On the other hand, small packet sizes lead to inefficient transmission due to the overhead caused by network protocols and error correcting codes, if applicable. This tradeoff can be influenced in favor of longer packet sizes through forward error correction (FEC) codes, which provide error resiliency in wireless communication. This advantage, however, is provided at the cost of increased energy consumption and latency due to encoding/decoding and transmission of longer packets as investigated in [19].

Another important source of energy consumption is collisions that occur in the high density WSN. While the MAC scheme in use affects the collision rate of the network, here, we show that the packet size has also an important effect on collisions in WSN.

Generally, the packet size is assumed to have negative effect on collisions. In cellular and ad hoc networks, it is accepted that longer packet sizes increase the collision rate of the network. This is due to the fact that a MAC layer frame size is determined assuming a fixed and saturated traffic load. In WSN, however, the generated traffic is directly related to the physical phenomenon that is being sensed and the properties of the onboard sensors. Since the existing onboard sensors provide data rates in the orders of $1-5 \mathrm{bps}$, the traffic generation rate is much less than the channel capacity, which is $250 \mathrm{kbps}$ for MicaZ nodes. Consequently, saturation traffic cannot be assumed and hence, the collision rates are closely related to the packet size.

For medium access, we assume that a node performs carrier sense mechanism to assess the availability of the channel and transmits a packet thereafter. Note that if a reservation-based protocol is used, collisions may not occur. In those cases, the remainder of our framework still applies. Many work focus on the investigation of medium access performance of carrier sense mechanisms [5], [6], [8], [16]. Without loss of generality, here, we refer to the work in [16]. Note that our contribution is not to produce yet another analysis of carrier sense mechanism. Rather, we aim to illustrate the impact of packet size on collision rate in WSN, which, to the best of our knowledge, has not been performed before.

We first illustrate the formulation in [16] and then present our results. Accordingly, a successful allocation of the channel depends on both successful carrier sense and the fact that the transmission encounters no collisions. The probability of successful carrier sense, $p_{c s}$, can be denoted as follows [16]:

$$
p_{c s}=1-\left(1-p_{c f}\right)^{K+1}
$$

where $K$ is the number of re-sensings allowed for one transmission and $p_{c f}$ is the probability of sensing the channel free, which is given by:

$$
p_{c f}=e^{-\lambda_{n e t}\left(\tau_{c s}+T_{\text {comm }}\right)}
$$

where $\tau_{c s}$ is the carrier sense period and $T_{\text {comm }}$ is the duration of a packet transmission. After a successful carrier sense, a collision can only occur if another node transmits during the vulnerable period of $\tau_{c s}$. As a result, the probability of no collisions, $p_{\text {coll }}$, is given by

$$
p_{\text {coll }}=e^{-\lambda_{\text {net }} \tau_{c s}}
$$

The term $\lambda_{\text {net }}$ that appears both in (2) and (3) refers to the overall traffic that is generated by all the nodes inside the transmission range of a node, which is given by

$$
\lambda_{\text {net }}=\lambda \frac{p_{c s}}{p_{\text {comm }}}\left(1-\left(1-p_{\text {comm }}\right)^{L+1}\right),
$$

where $\lambda$ is the total generated traffic in the transmission range of a node and $p_{\text {comm }}$ is the probability of successful transmission. Accordingly, the probability that a node can successfully acquire the channel is given by $p_{c s} p_{\text {coll }}$, which can be found by solving equations (1), (3), and (4).

The effect of packet size on the MAC failure rate, i.e., 1 $p_{c s} p_{\text {coll }}$, can be observed clearly through (2) and (3). First, the probability of sensing the channel free, $p_{c f}$, depends on the duration of a packet transmission, $T_{\text {comm }}$. Longer packet sizes decrease this probability since a node will acquire the channel for a longer time. Second, the packet size, $l_{D}$, impacts the generated traffic rate $\lambda$, which affects both the probability of no collisions, $p_{\text {coll }}$, and the probability of successful carrier sense, $p_{c s}$. 
The total generated packet rate, $\lambda$, depends on both the generated traffic rate and the size of the packet. Let us assume that the sensor node has an average sampling rate of $b$ bits/s. Denoting the length of the packet payload as $l_{D}$, on the average, the packet generation rate of a node $i$ is $\lambda_{i i}=b / l_{D}$ pkts/s. Since a node will also relay packets from other nodes to the sink, the packet transmission rate of a node is higher than this value. If a routing scheme that equally shares the network load among nodes is considered, on the average, the packet transmission rate of a node is $\lambda_{i}=c_{i} \lambda_{i i}$, where $c_{i}>1$. Consequently, $\lambda$ in (4) is given by $\lambda=\sum_{i=1}^{M} \lambda_{i}$, where the number of nodes that are in the transmission range of a node is given by $M-1$. It can be clearly seen that an increase in packet size directly affects the rate of channel access attempts and hence the traffic on the channel. The traffic rate affects both the probability of successful carrier sense, $p_{c s}$, and the probability of no collisions, $p_{\text {coll }}$. The MAC failure rate, $1-p_{c s} p_{\text {coll }}$, which is found using (1), (2), (3), and (4) as a function of payload length for different values of $M$ and $b=5$ bits/s for a WSN is given in Fig. 1. It can be observed that increase in payload length decreases the MAC failure rate.

The results of this analysis reveal that longer packet sizes are favorable in WSN when collisions are considered alone. This is motivated by the cross-layer interdependency of generated traffic and the packet size in WSN. Although these results are significant, an overall assessment of the packet size on the network performance is required. Especially the energy consumption of the overall communication needs to be investigated to provide a complete solution for packet size optimization in WSN. In the following sections, we provide a comprehensive analysis of packet size based on the observations in this section.

\section{PACKet Size Optimization Framework}

We formalize our optimization framework based on endto-end performance metrics. More specifically, the communication performance metrics such as energy consumption, throughput, latency, and reliability are derived for a flow that is generated at a sensor node and transmitted to the sink through a multi-hop path. We formalize our optimization solution by using three different objective functions. Each objective function highlights a different aspect of communication in WSN and can be selected according to the requirements of the application in use. Furthermore, we investigate the effects of end-to-end latency and reliability constraints that may be required by a particular application. As a result, a flexible optimization framework is developed to determine the optimal packet size in WSN. Next, we define these objective functions.

\section{A. Objective Functions}

We define three optimization metrics as packet throughput, energy per useful bit, and resource utilization.

Definition 1: Packet throughput:

$$
\mathcal{U}_{\text {tput }}=\frac{l_{D}\left(1-P E R_{e 2 e}\right)}{T_{\text {flow }}},
$$

where $l_{D}$ is the payload length, $P E R_{e 2 e}$ is the end-to-end packet error rate, which considers the entire packet including header and trailer, and $T_{\text {flow }}$ is the end-to-end latency, which is the time spent between a packet is generated at a sensor and received at the sink through the multi-hop route. The packet throughput function considers the end-to-end packet success rate and the end-to-end delay to transmit a packet of payload $l_{D}$

\section{Definition 2: Energy per useful bit:}

$$
\mathcal{U}_{\text {eng }}=\frac{E_{\text {flow }}}{l_{D}\left(1-P E R_{e 2 e}\right)}
$$

where $E_{\text {flow }}$ is the end-to-end energy consumption to transport a packet from a source to a destination. The utility function, $\mathcal{U}_{\text {eng }}$, is the energy consumption for useful bit between a particular node and the sink. Minimizing $\mathcal{U}_{\text {eng }}$ results in optimal packet size values that achieve high energy efficiency.

Minimizing energy consumption is the main goal in WSN. However, $\mathcal{U}_{e n g}$ in (6) does not consider the delay associated with the optimal value of the packet size that minimizes this function. Since end-to-end delay is also important in WSN, the third objective function considers the energy-delay product consumed for useful bit, which is referred to as resource utilization:

\section{Definition 3: Resource utilization:}

$$
\mathcal{U}_{\text {res }}=\frac{E_{\text {flow }} T_{\text {flow }}}{l_{D}\left(1-P E R_{e 2 e}\right)}
$$

Minimizing $\mathcal{U}_{\text {res }}$ leverages the tradeoff between energy consumption and latency. We believe this utility function serves best for practical implementation of WSN, especially for delay sensitive traffic.

In the remainder of this section, we present the derivation of the terms in the utility functions, $\mathcal{U}_{\text {tput }}$ in (5), $\mathcal{U}_{\text {eng }}$ in (6), and $\mathcal{U}_{\text {res }}$ in (7). For the derivation, we consider a channel-aware routing algorithm, where the next hop is determined according to the received signal to noise ratio (SNR) of a packet sent from a specific node $i$ at a distance $D$ from the sink [19]. Among the neighbors of $i$, the neighbor, $j$, that is closest to the sink and with SNR value, $\psi_{j}>\psi_{T h}$ is selected as the next hop, where $\psi_{T h}$ is the received SNR threshold. Note that this approach can be implemented using a cross-layer approach as in [3] or through signaling [17]. The medium access is performed through RTS-CTS-DATA exchange in addition to ACK and retransmissions for ARQ. To illustrate specific results for FEC codes, we consider block codes due to their energy efficiency and lower complexity compared to convolutional codes [15], [18]. We consider a block code, which is represented by $(n, k, t)$, where $n$ is the block length, $k$ is the payload length, and $t$ is the error correcting capability in bits. In our analysis, we use extended $\mathrm{BCH}$ codes, which enable the evaluation of the effect of error correction capability, $t$, by fixing the block length, $n=128$. However, other FEC schemes can also be used in our framework.

First, we explain the channel model used for the analysis. Then, the general analysis model and the derivation of each component is given.

\section{B. Channel Model}

For our derivations, we use the log-normal channel model, which has been experimentally shown to model the low power communication in WSN accurately [20]. In this model, the received power at a receiver at distance $d$ from a transmitter is given by

$$
P_{r}(d)=P_{t}-P L\left(d_{0}\right)-10 \eta \log _{10}\left(\frac{d}{d_{0}}\right)+X_{\sigma},
$$

where $P_{t}$ is the transmit power in $\mathrm{dBm}, P L\left(d_{0}\right)$ is the path loss at a reference distance $d_{0}$ in $\mathrm{dB}, \eta$ is the path loss exponent, and $X_{\sigma}$ is the shadow fading component, with $X_{\sigma} \sim \mathcal{N}(0, \sigma)$. 
Moreover, the SNR at the receiver is given by $\psi(d)=P_{r}(d)-$ $P_{n}$ in $\mathrm{dB}$, where $P_{n}$ is the noise power in $\mathrm{dBm}$.

Considering the shadow fading component, $X_{\sigma}$, the probability that the received SNR, $\psi_{j}$, of the channel between two nodes $n_{i}$ and $n_{j}$ that are at a distance $d_{(i, j)}$ is above some threshold, $\psi_{T h}$, is

$$
\mathrm{P}\left\{\psi_{j}>\psi_{T h}\right\}=Q\left(\frac{\beta\left(d_{(i, j)}, \psi_{T h}\right)}{\sigma}\right),
$$

where

$$
\beta\left(d, \psi_{T h}\right)=\psi_{T h}+P_{n}-P_{t}+P L\left(d_{0}\right)+10 \eta \log _{10}\left(\frac{d}{d_{0}}\right)
$$

and $Q(x)=1 / \sqrt{2 \pi}\left(\int_{x}^{\infty} e^{-\left(t^{2} / 2\right)}\right) d t$. Based on this channel model, the end-to-end energy consumption for a packet, $E_{\text {flow }}$, will be derived as will be shown in the following section.

\section{End-to-End Energy Consumption}

The end-to-end energy consumption, $E_{\text {flow }}$, of a packet sent from a node at a distance $D$ from the sink is derived based on the model in [19], which is extended here to accommodate medium access collisions according to the discussion in Section III. The end-to-end energy consumption for a packet, $E_{\text {flow }}$, is given as follows:

$$
E_{\text {flow }}=\mathrm{E}\left[E_{h}\right]\left(\frac{D-R_{i n f}}{\mathrm{E}\left[d_{h}\right]}+1\right),
$$

where $\mathrm{E}\left[E_{h}\right]$ is the expected energy consumption per hop, $D$ is the distance between the source node and the sink, $R_{\text {inf }}$ is the approximated transmission range of a node, and $\mathrm{E}\left[d_{h}\right]$ is the expected hop distance. Accordingly, the expected hop distance is given by

$$
\mathrm{E}\left[d_{h}\right]=\rho \delta \int_{\gamma_{\min }}^{D} \int_{-\alpha_{\gamma}}^{\alpha_{\gamma}} \gamma d_{(i, j)} Q\left(\frac{\beta}{\sigma}\right) e^{-M\left(1-p_{k}\right)} d \alpha d \gamma,
$$

where $\rho$ is the node density, $\delta$ is the duty cycle value, $\gamma$ is the distance between the next hop and the sink, $d_{(i, j)}$ is the distance between the source node and the next hop, $\beta$ is as given in (10), and $e^{-M\left(1-p_{k}\right)}$ is the probability that the next hop is at least at a distance $\gamma$ from the sink.

Similarly, the expected energy consumption per hop, $\mathrm{E}\left[E_{h}\right]$, in (11) can be found as

$$
\mathrm{E}\left[E_{h}\right]=\rho \delta \int_{\gamma_{\min }}^{D} \int_{-\alpha_{\gamma}}^{\alpha_{\gamma}} \gamma \mathrm{E}\left[E_{j}\right] Q\left(\frac{\beta}{\sigma}\right) e^{-M\left(1-p_{k}\right)} d \alpha d \gamma,
$$

where $\mathrm{E}\left[E_{j}\right]$ is the expected energy consumption, which is given as

$$
\mathrm{E}\left[E_{j}\right]=\int_{\psi_{T h}}^{\infty} E_{c o m m}\left(\psi, d_{(i, j)}\right) f_{\Psi}\left(\psi, d_{(i, j)}\right) d \psi .
$$

In (14), $f_{\Psi}\left(\psi, d_{(i, j)}\right)=1 /(\sigma \sqrt{2 \pi}) e^{\frac{-\beta^{2}}{2 \sigma^{2}}}$ is the pdf of the SNR. Moreover $E_{\text {comm }}\left(\psi, d_{(i, j)}\right)$ is the energy consumption for communication between nodes $i$ and $j$ given that they are at a distance $d_{(i, j)}$ with a SNR value of $\psi$ at node $j$, which has three components as given by

$$
E_{\text {comm }}=E_{T X}+E_{R X}+E_{n e i g h},
$$

where $E_{T X}$ is the energy consumed by the node transmitting the packet, $E_{R X}$ is the energy consumed by the node receiving the packet, and $E_{n e i g h}$ is the energy consumed by the neighbor nodes [19]. The framework in [19] is improved by considering the affect of collision rate on the success of the transmission of each packet. Hence, the energy consumption for transmitter node, $E_{T X}$, for ARQ and FEC are shown in (16) and (17), respectively, where the effects of collisions are also reflected. The first term in each equation is the retransmission rate, where $p_{c s}$ is the probability of successful carrier sense given in (1), $p_{c o l l}$ is the probability of no collisions given in (3), and $p_{s}^{C}$ and $p_{s}^{D}$ are the probability of success for control and data packets, respectively. Similarly, the values for $E_{R X}$ and $E_{n e i g h}$ can be found, which are not shown here for space limitations. Once the $E_{\text {comm }}$ in (15) is found, the end-to-end energy consumption, $E_{\text {flow }}$, is calculated using (12-14) in (11). Moreover, the endto-end latency, $T_{\text {flow }}$, and the end-to-end packet error rate, $P E R_{e 2 e}$, are also found using the similar approach in [19] and the extension discussed above. Next, we formalize our optimization problem for packet size optimization in WSN.

\section{OPTIMIZATION RESULTS}

It can be observed from (11-15) that the energy consumption of a flow is mainly affected by the packet size and the SNR threshold, $\psi_{T h}$. This is also true for the end-to-end packet error rate and the end-to-end delay. Consequently, these two parameters affect the utility functions in (5-7). In Fig. 2(a), the energy per useful bit is shown as a function of payload length $l_{D}$ for three different values of SNR threshold $\psi_{T h}$ for ARQ. It is evident that the optimum value for packet size for a specific objective function depends on the routing decisions and hence $\psi_{T h}$.

The effect of the SNR threshold value $\psi_{T h}$ can be explained as follows: $\psi_{T h}$ controls the minimum quality of the wireless channel at each hop since the routes are constructed according to this value. Moreover, the average hop length increases for a lower $\psi_{T h}$ value [19]. This has two consequences in overall energy consumption of the communication: If low quality links are chosen, the energy consumption may increase due to retransmissions or packet drops. On the other hand, since longer hops are constructed, the overall energy consumption can still be decreased. For a low $\psi_{T h}$ value, low quality links may be chosen, which necessitates smaller packet sizes to maintain an acceptable reliability. However, this causes inefficiency due to increased affect of header and trailer of the packet and may decrease the energy efficiency. As a result, packet size optimization is significantly affected by the routing decisions. Hence, choice of the SNR threshold value, $\psi_{T h}$, determines the optimum packet size. Hence, in our optimization problem, our goal is to find both $\psi_{T h}$ and the packet size, $l_{D}$. Consequently, our optimization problems become:

$\mathbf{P}_{\text {max }}^{\text {tput }}$ : Packet throughput maximization

$$
\begin{array}{rc}
\text { Given }: & D, \eta, \sigma, n, k, t \\
\text { Find }: & \psi_{T h}, l_{D} \\
\text { Maximize }: & \mathcal{U}_{\text {tput }}=\frac{l_{D}\left(1-P E R_{e 2 e}\right)}{T_{\text {flow }}}
\end{array}
$$

$\mathbf{P}_{\text {min }}^{\text {eng }}$ : Energy consumption per useful bit minimization

$$
\begin{array}{rc}
\text { Given }: & D, \eta, \sigma, n, k, t \\
\text { Find }: & \psi_{T h}, l_{D} \\
\text { Minimize }: & \mathcal{U}_{\text {eng }}=\frac{E_{f l o w}}{l_{D}\left(1-P E R_{e 2 e}\right)}
\end{array}
$$




$$
\begin{aligned}
E_{T X}^{A R Q}= & \frac{p_{\text {cs }}}{1-p_{\text {cs }}+p_{\text {cs }} p_{\text {coll }}\left(p_{s}^{C}\right)^{3} p_{s}^{D}}\left\{E_{\text {sense }}+E_{t x}^{C}+p_{\text {coll }} p_{s}^{C} E_{r x}^{C}+\left(1-p_{\text {coll }} p_{s}^{C}\right) E_{t / o}^{C}+p_{\text {coll }}\left(p_{s}^{C}\right)^{2} E_{t x}^{D}\right. \\
& \left.+p_{\text {coll }}\left(p_{s}^{C}\right)^{2} p_{s}^{D} E_{r x}^{C}+p_{\text {coll }}\left(p_{s}^{C}\right)^{2}\left(1-p_{s}^{D}\right) E_{t / o}^{D}\right\} \\
E_{T X}^{F E C}= & \frac{p_{c s}}{1-p_{\text {cs }}+p_{\text {cs }} p_{\text {coll }}\left(p_{s}^{C}\right)^{2}}\left\{E_{\text {sense }}+E_{\text {tx }}^{C}+p_{\text {coll }} p_{s}^{C}\left(E_{r x}^{C}+E_{\text {dec }}^{C}\right)+\left(1-p_{\text {coll }} p_{s}^{C}\right) E_{t / o}^{C}\right\} \\
& +p_{\text {cs }} p_{\text {coll }}\left(p_{s}^{C}\right)^{2} E_{t x}^{D}
\end{aligned}
$$

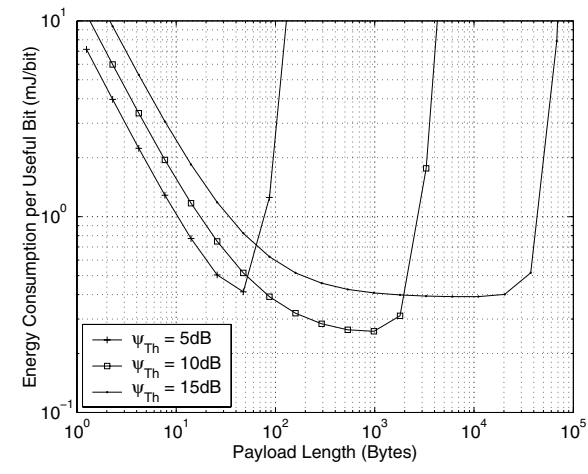

(a)

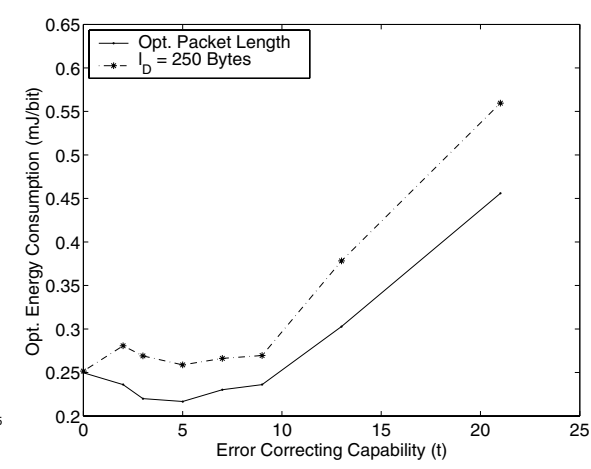

(b)

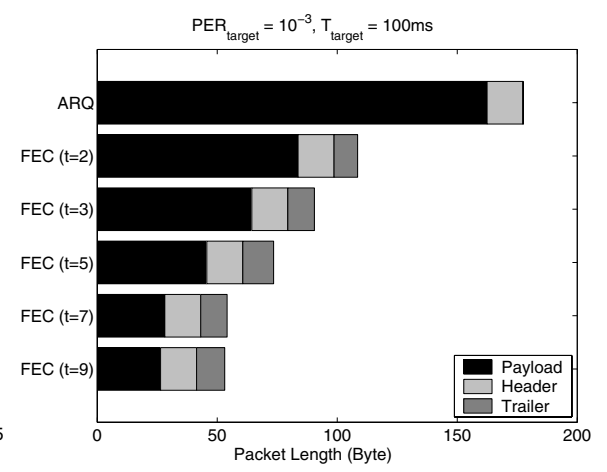

(c)

Fig. 2. Energy per useful bit (6) vs. payload length for (a) ARQ and (b) Energy consumption per bit with optimum packet length and $l_{D}=250$ bytes., and (c) optimal packet length for different error control techniques.

\section{$\mathbf{P}_{\text {min }}^{\text {res }}:$ Resource utilization minimization}

$$
\begin{array}{rc}
\text { Given }: & D, \eta, \sigma, n, k, t \\
\text { Find }: & \psi_{T h}, l_{D} \\
\text { Minimize }: & \mathcal{U}_{\text {res }}=\frac{E_{\text {flow }} T_{\text {flow }}}{l_{D}\left(1-P E R_{e 2 e}\right)}
\end{array}
$$

The optimum values of $l_{D}$ and $\psi_{T h}$ have been found using the optimization toolbox of MATLAB. In Table I, the results are shown for the three optimization problems, $\mathbf{P}_{\max }^{\text {tput }}, \mathbf{P}_{\min }^{\text {eng }}$, and $\mathbf{P}_{\text {min }}^{\text {res }}$. The columns $l_{D}, \psi_{T h}, \mathcal{U}_{\text {eng }}, T_{\text {flow }}, \mathcal{U}_{\text {tput }}$, and $p_{\text {flow }}$ refer to optimal payload length, optimal SNR threshold, energy consumption per useful bit, end-to-end latency, packet throughput, and end-to-end success rate $\left(1-P E R_{e 2 e}\right)$, respectively. When the throughput maximization problem, $\mathbf{P}_{\max }^{\text {tput }}$, is concerned, ARQ scheme with a payload length of 152 bytes achieves the highest throughput, $\mathcal{U}_{\text {tput }}$.

The energy per bit minimization problem, $\mathbf{P}_{\text {min }}^{\text {eng }}$, results in favor of FEC schemes which can accommodate longer packet sizes without affecting the energy efficiency. However, very long packet sizes are determined for this problem. This is due to the fact that longer packet sizes are more efficient when a sufficient link quality is guaranteed. Nevertheless, optimal packet values may not be feasible in current wireless sensor nodes. As an example, the ZigBee standard defines 250 byte as the maximum packet length [11]. In Fig. 2(b), the optimal energy consumption per bit is shown for both the optimum $l_{D}$ values and 250 byte packet size for various error correcting capability, $t$. The values for $t=0$ correspond to the ARQ case. It is evident that when high packet lengths can not be accommodated, energy efficiency decreases as much as $20 \%$.

The results for $\mathbf{P}_{\text {min }}^{\text {res }}$ show how the proposed resource utilization metric leverages energy consumption and end-to-end latency performance. By compromising from energy consumption per bit, $\mathcal{U}_{\text {eng }}$, (2-fold increase), end-to-end latency, $T_{\text {flow }}$, can be significantly decreased (15 times decrease) leading to optimal payload length, $l_{D}$, values in the range of 25-50 bytes.
In this case, when energy efficiency is concerned, FEC with $t=5$ and payload length of $l_{D}=53$ bytes is optimal. If throughput, $\mathcal{U}_{\text {tput }}$, and end-to-end success rate, $p_{\text {flow }}$ are also important, ARQ scheme with packet length of $l_{D}=25$ bytes is the optimal value with a slight increase in energy consumption per bit, $\mathcal{U}_{\text {eng }}$.

Certain WSN applications that focus mainly on real-time monitoring require strict end-to-end latency and success rate (reliability) constraints. Based on these observations, we develop an optimization framework for energy minimization subject to delay and reliability constraints. The optimization problem with delay and reliability constraints is cast as follows:

$\mathbf{P}_{\text {min, }{ }^{e n}}^{\text {: }}$ : Energy consumption per useful bit minimization with delay and reliability constraints

$$
\begin{array}{rc}
\text { Given }: & D, \eta, \sigma, n, k, t, P_{\text {target }}, T_{\text {target }} \\
\text { Find : } & \psi_{T h}, l_{D} \\
\text { Minimize : } & \mathcal{U}_{\text {eng }}=\frac{E_{\text {flow }}}{l_{D}\left(1-P E R_{e 2 e}\right)} \\
\text { Subject to : } & T_{\text {flow }} \leq T_{\text {target }} \\
& P E R_{e 2 e} \leq P_{\text {target }}
\end{array}
$$

where $T_{\text {target }}$ and $P_{\text {target }}$ are end-to-end delay and end-to-end packet error rate constraints, respectively.

Considering an end-to-end packet error rate of $P_{\text {target }}=$ $10^{-3}$ and end-to-end latency of $T_{\text {target }}=100 \mathrm{~ms}$, in Fig. 2(c), the optimum packet sizes including the header and trailer for each error control technique are shown. When end-to-end delay, $T_{\text {flow }}$, and success rate, $p_{\text {flow }}$, is important, our results show that ARQ with optimal payload length of $l_{D}=162$ bytes result in optimal performance.

\section{PACKet Size Optimization in Wireless UNDERWATER AND UNDERGROUND SENSOR NETWORKS}

In this section, we extend our analysis of packet size optimization for underwater and underground sensor networks. 
TABLE I

OPTIMAL $l_{D}$ AND $\psi_{T h}$ VALUES

\begin{tabular}{|l|l||c|c||c|c|c|c|}
\hline Problem & ECC Type & $l_{D}($ byte $)$ & $\psi_{T h}(d B)$ & $\mathcal{U}_{\text {eng }}(\mathrm{mJ} /$ bit $)$ & $T_{\text {flow }}(\mathrm{ms})$ & $\mathcal{U}_{\text {tput }}(\mathrm{kbps})$ & $p_{\text {flow }}$ \\
\hline \multirow{5}{*}{$\mathbf{P}_{\text {max }}^{\text {tput }}$} & ARQ (N=4) & 152 & 7.5 & 0.2659 & 76.7 & 15.8 & 0.9996 \\
& FEC (t=5) & 1103 & 4.5 & 0.2204 & 1419 & 6.1 & 0.9813 \\
& FEC (t=7) & 546 & 3 & 0.2450 & 944 & 4.5 & 0.9674 \\
& FEC (t=9) & 1133 & 2.5 & 0.2389 & 2482 & 3.6 & 0.9840 \\
\hline \multirow{5}{*}{$\mathbf{P}_{\text {min }}^{\text {eng }}$} & ARQ (N=4) & 473 & 9 & 0.2497 & 248.3 & 15.2 & 0.9994 \\
& FEC (t=5) & 4933 & 5 & 0.2167 & 6521 & 5.9 & 0.9849 \\
& FEC (t=7) & 2915 & 3.5 & 0.2302 & 5106 & 4.4 & 0.9719 \\
& FEC (t=9) & 5342 & 3 & 0.2360 & 12019 & 3.5 & 0.9910 \\
\hline \multirow{5}{*}{$\mathbf{P}_{\text {min }}^{\text {res }}$} & ARQ (N=7) & 25 & 5 & 0.5190 & 17.5 & 11.2 & 0.9998 \\
& FEC (t=5) & 53 & 3.5 & 0.5028 & 93.9 & 4.4 & 0.9797 \\
& FEC (t=7) & 39 & 2 & 0.5917 & 90.7 & 3.2 & 0.9495 \\
& FEC (t=9) & 26 & 1 & 0.7496 & 85.4 & 2.3 & 0.9437 \\
\hline
\end{tabular}

Considering the unique properties of these networks compared to terrestrial wireless sensor networks, the optimization framework is extended and the optimal packet sizes for each of these networks are found. We first present the channel models for these networks and discuss the unique properties of these networks. Then, the results for our optimization problems are provided for these challenged environments.

\section{A. Underwater Channel Model}

Underwater Acoustic Sensor Networks (UW-ASN) are characterized by acoustic communication channel [2], which is characterized by the Urick path loss formula which is given below:

$$
T L(d, f)=\chi \cdot \log (d)+\alpha(f) \cdot d+A
$$

where the path loss, $T L(d, f)$, is shown in $\mathrm{dB}$ as a function of internode distance $d$ and operating frequency $f$. The term $\chi$ is the geometric spreading which can be spherical for deep water and cylindrical for shallow water. The last term $A$ is the transmission anomaly and accounts for the degradation of the acoustic intensity caused by multiple path propagation, refraction, diffraction, and scattering of sound. Moreover, the propagation delay in underwater acoustic channel varies between $1460 \mathrm{~m} / \mathrm{s}$ and $1520 \mathrm{~m} / \mathrm{s}$.

Moreover, we model the randomness in the channel using the Rayleigh fading channel model [10], where the envelope of the signal is modeled as a Rayleigh distributed random variable, $\alpha$. Consequently, the signal-to-noise ratio (SNR) of the channel is given in $\mathrm{dB}$ in underwater acoustic channels as

$$
\psi_{0, d B}(d, f)=S L_{d B \text { re } и \text { Pa }}-T L_{d B}(d, f)-N L_{d B \text { re } и \text { Р } a}
$$

where $S L_{d B \text { re uPa }}$ and $N L_{d B \text { reuPa }}$ are the signal level at the transmitter and the noise level given in $\mathrm{dB}$ with reference to $\mu P a$. Then, $E_{b} / N_{o}=\psi B_{N} / R$, where $\psi=10^{\psi_{d B}}(d, f) / 10$, $B_{N}$ is the noise bandwidth, and $R$ is the data rate. The signal level, $S L$, in (33) can be related to the intensity, $I_{t}$ and, hence, the transmit power, $P_{t}$ of the transceiver as follows:

$$
I_{t}=\frac{P_{t}}{2 \pi 1 m H}, S L=10 \log \left(\frac{I_{t}}{0.67 \times 10^{-18}}\right),
$$

where $P_{t}$ is the transmit power in Watts, and $H$ is the depth in $m$.

An important observation about underwater communication is the effect of surface reflected rays as shown through ex- periments in [7]. More specifically, it has been observed that the received signal follows a 2-path Rayleigh model, where the direct path and the surface reflected path contributes to the received signal strength. Moreover, the signal from each path can be modeled as an independent Rayleigh distributed signal. Consequently, the bit error rate experienced at a node is a result of combination of these signals.

In order to apply our optimization framework developed in Section IV to the underwater channel, the bit error rate and the probability that the received signal to noise ratio (SNR) is higher than a threshold needs to be found. Since the 2-path Rayleigh channel model does not have a closedform expression for SNR distribution, we have performed simulations to find these values. In our simulations, 5000 independent pairs of Rayleigh distributed random variables are generated. The received signal strength for each location is then found for these independent trials. Consequently, the empirical cdf found as a result of these trials is used for (9). This value is used to find the expected bit error rate, energy consumption, and latency at each point in the simulations.

\section{B. Underground Channel Model}

The channel model for underground wireless communication has been developed in [13]. This model follows a 2path location-based Rayleigh fading channel model. While the details of this model can be found in [13], it is important to note that the path loss, $L_{p}$, in an underground environment is a function of the attenuation constant, $\alpha$, and the phase shifting constant, $\beta$, which depend on the volumetric water content (VWC) of the soil, its bulk density as well as the mass fractions of sand and clay. Consequently, the path loss is a function of soil properties as well as the volumetric water content of the soil. As shown in [13], if the sensors are buried at a depth less than $2 \mathrm{~m}$, i.e., low depth, the influence of the wave reflection by ground surface should be considered. Consequently, the total path loss of two-path channel model is given as follows:

$$
L_{f}(d B)=L_{p}(d B)-V_{d B},
$$

where $L_{p}$ is the path loss due to the single path and $V_{d B}$ is the attenuation factor due to the second path in $\mathrm{dB}$ as derived in [13]. Finally, the bit error rate (BER) is shown as $p_{b}=0.5 \operatorname{erfc}(\sqrt{\psi})$, where $\operatorname{erfc}(\cdot)$ is the error function and the SNR is given by $\psi=P_{t}-L_{f}-P_{n}$, where $P_{t}$ is the transmit power, $L_{f}$ is the total path loss given in (35), and 


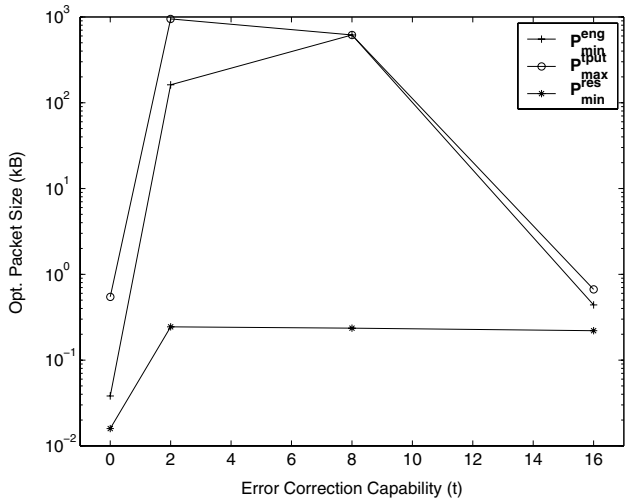

(a) Optimum Packet Size for Deep Water

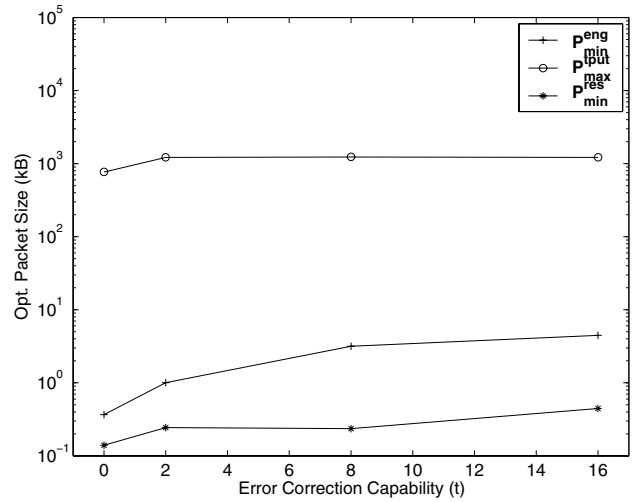

(b) Optimum Packet Size for Shallow Water

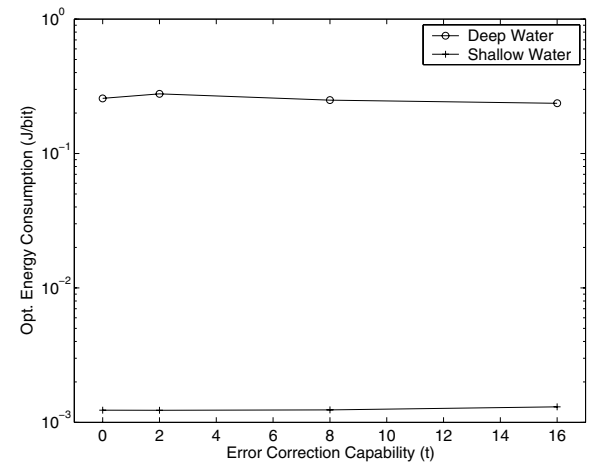

(c) Optimum Energy Consumption

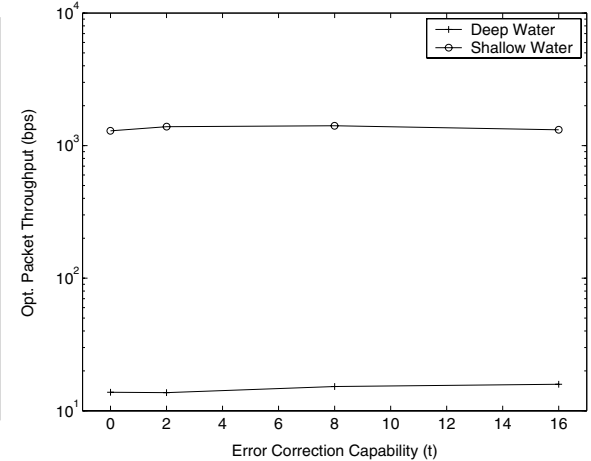

(d) Optimum Packet Throughput

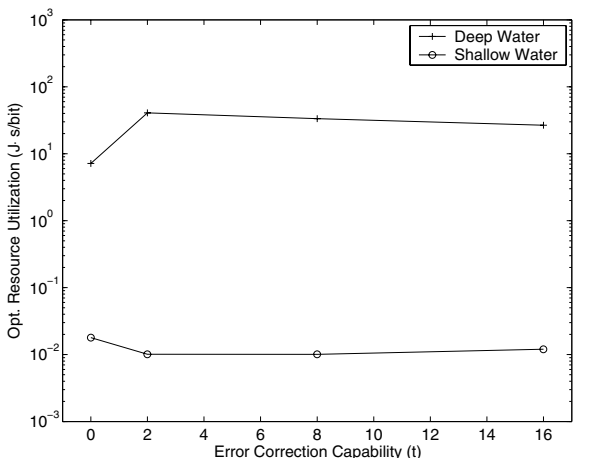

(e) Optimum Resource Utilization

Fig. 3. Optimization results for $\mathbf{P}_{\text {min }}^{\text {eng }}, \mathbf{P}_{\max }^{\text {tput }}$, and $\mathbf{P}_{\text {Min }}^{\text {eng }}$.

$P_{n}$ is the energy of noise, which is found to be $-103 \mathrm{dBm}$ [13]. Using simulations, the bit error rate and the probability that the received SNR is higher than a threshold are found similar to the underwater case. Next, we present the results of our packet size optimization for underwater and underground sensor networks.

\section{Results}

In this section, we present the results for packet size optimization for underwater and underground environment. More specifically, the optimum packet size is found by solving three different optimization problems, i.e., $\mathbf{P}_{\text {min }}^{\text {eng }}, \mathbf{P}_{\text {max }}^{\text {tput }}$, and $\mathbf{P}_{\text {Min }}^{\text {eng }}$ defined in Section IV. Furthermore, we present the results for constrained optimization problem, where the optimum packet size is determined subject to reliability and latency constraints. Our results focus on two major communication environments, i.e., underwater (Section VI-C1) and underground (Section VI-C2). For the underwater case, we consider a deep water network, where the sensors are deployed close to the ocean bottom. For this case, we use the two-ray underwater channel model explained in Section VI-A. These networks are used for ocean bottom measurements and surveillance. For the shallow water case, we consider a network deployed close to the surface of the water, where reflections from the sea surface needs to be considered. Finally, we investigate the packet size optimization problem in wireless underground sensor networks according to the channel model presented in Section VI-B and [13]. In particular, we present the effects of volumetric water content on the optimum packet size.

1) Wireless Underwater Sensor Networks: Next, we present the results for packet size optimization in deep water and shallow water environment. It is important to note that the differences between propagation characteristics for deep and shallow water result in significantly different optimum packet lengths for these environments.

a) Deep Water Environment: The optimization results for this case are shown in Figs. 3. In Fig. 3(a), the optimum packet size for the three optimization problems, $\mathbf{P}_{\min }^{\text {eng }}, \mathbf{P}_{\text {max }}^{\text {tput }}$, and $\mathbf{P}_{M i n}^{e n g}$ are shown as a function of the error correction capability, $t$. Note that $t=0$ corresponds to the ARQ scheme. The corresponding optimum values for energy consumption, throughput, and resource utilization are also shown in Fig. 3(c) - Fig. 3(e). For each problem, the ARQ scheme can accommodate smaller packet sizes than the FEC codes. As an example, the optimum packet size for the throughput maximization problem is 547 bytes for ARQ scheme and 616 Kbytes for RS $(255,239,8)$ code. Despite this significant increase in optimum packet size for the RS $(255,239,8)$ code, the throughput is also increased $9 \%$ compared to the ARQ scheme. It can be observed from Fig. 3(c) that the energy consumed per successfully received bit is very similar $(0.25 \mathrm{~J} / \mathrm{bit}$ for ARQ and $0.24 \mathrm{~J} / \mathrm{bit}$ for RS $(255,239,8)$ ), which shows that forward error correction codes provide significant data transmission capability without hampering the energy consumption in deep water environment.

b) Shallow Water Environment: Shallow water communication is characterized by a lower propagation loss compared to deep water because of both the cylindrical spreading and reflection from the water surface. The optimum packet size values for each of the optimization problems are shown in Fig. 3(b). Compared to the deep water environment, in shallow water environment, channel errors are not severe and hence, the ARQ scheme also results in optimum packet size values 


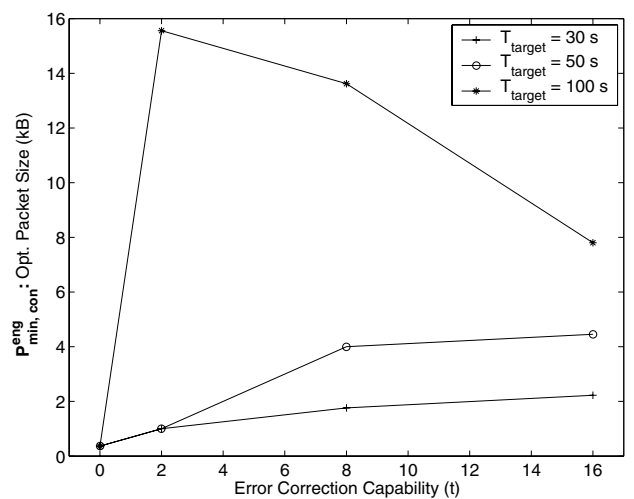

(a) Optimum Packet Size for $\mathbf{P}_{m i n, c o n}^{e n g}$

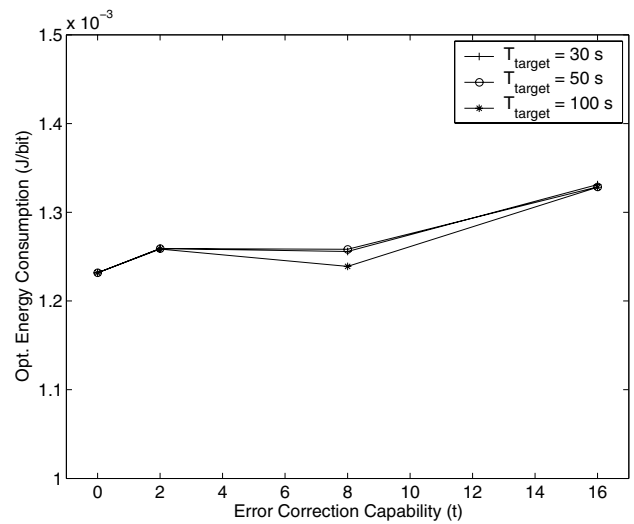

(b) Optimum Energy Consumption

Fig. 4. Constrained optimization results as a function of error correction capability, $t$, for different delay bounds in shallow water.

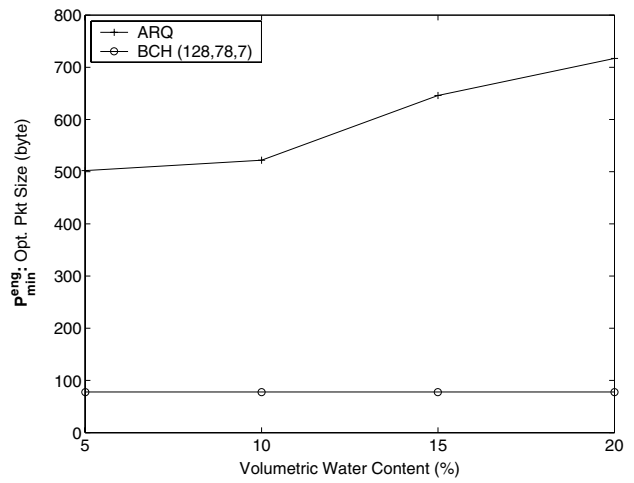

(a) Optimum packet size

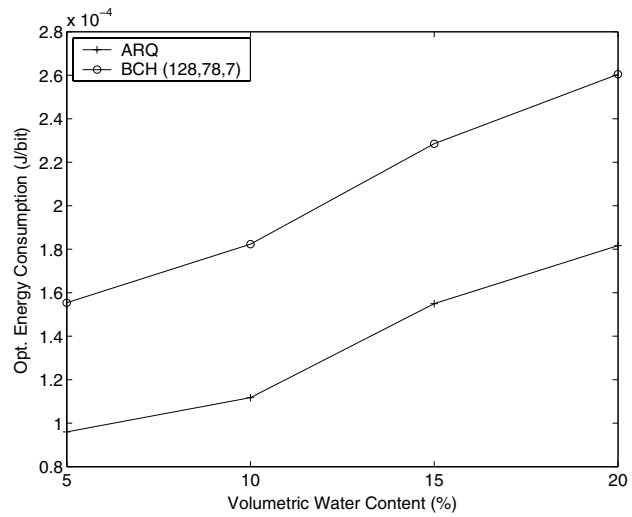

(b) Optimum energy consumption

Fig. 5. Optimization results for $\mathbf{P}_{m i n}^{e n g}$ in underground environment as a function of volumetric water content.

comparable to the FEC schemes. Moreover, as shown in Fig. 3(c) and Fig. 3(d), RS $(255,251,2)$ code is the most energy efficient and leads to highest throughput. Moreover, it is observed from Fig. 3(e) that RS codes are more resource efficient than the ARQ schemes.

We also present the results for the constrained optimization problem, $\mathbf{P}_{\text {min,con }}^{\text {eng }}$, in Fig. 4(a) and Fig. 4(b), where the optimal packet size and the resulting energy consumption are shown, respectively. For these results, we use $P_{\text {target }}=10^{-3}$ and three different values for the end-to-end delay bound. An important result is the significant decrease in optimum packet size. Since the end-to-end delay is bounded, packet sizes between 2-16 bytes are optimal depending on the error correction capability. While increasing the end-to-end delay increases the optimum packet size for the RS codes, the energy consumption is slightly decreased for larger $T_{\text {target }}$. Moreover, the optimum packet size for the ARQ scheme is not affected by the delay constrained.

2) Wireless Underground Sensor Networks: As we have investigated in [13], the underground communication is characterized by the composition as well as the volumetric water content of the soil. Consequently, we investigate the effects of volumetric water content on optimal packet size in underground sensor networks. We consider the ARQ scheme and BCH $(128,78,7)$ for our results. Moreover, we use the typical operation parameters for the Mica2 nodes [9], which are shown to be feasible for wireless underground sensor networks at frequencies $400 \mathrm{MHz}$ [13].
The effect of volumetric water content on energy consumption is shown in Figs. 5. The change in optimum packet size for energy consumption minimization is shown in Fig. 5(a). Increase in volumetric water content results in higher packet sizes for the energy consumption minimization problem, where the optimum energy consumption also increases for higher values of volumetric water content.

Finally, in Fig. 6, the results of the constrained optimization problem for the underground environment are shown. More specifically, the optimum packet sizes as well as the optimum energy consumption and packet throughput associated with them are shown as a function of end-to-end delay bound for different burial depth and volumetric water content (VWC) values. In Fig. 6(a), it is shown that the optimum packet size increases with increasing delay bound. Moreover, an increase in volumetric water content decreases the optimum packet size. Furthermore, this has an important effect on both energy consumption and packet throughput. In particular, increasing the volumetric water content from $5 \%$ to $20 \%$ increases energy consumption by $60 \%$ and decreases packet throughput by $37 \%$. This significant dependance on the volumetric water content necessitates the communication protocols be tailored to account for changes in the water content of the soil. An important observation is that relaxing the end-to-end delay bound cannot provide lower energy consumption higher than a specific value, i.e., 5 s. More specifically, the optimum energy consumption stays constant above this value of the end-to-end delay constraint. 


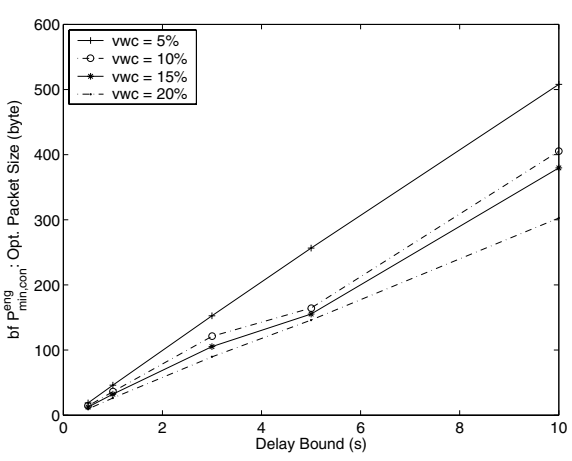

(a) Optimum Packet Size for $\mathbf{P}_{\min , \text { con }}^{\text {eng }}$

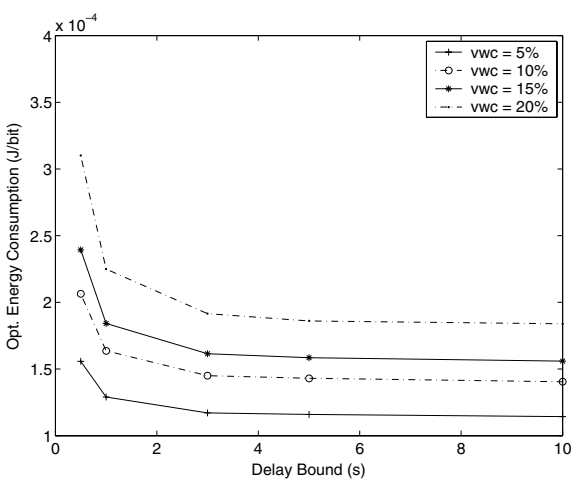

(b) Optimum Energy Consumption

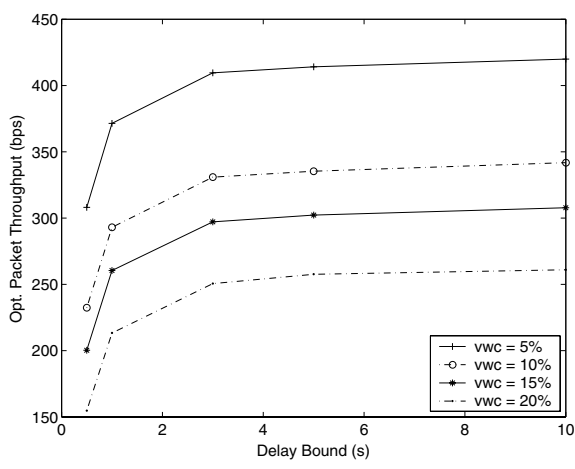

(c) Optimum Packet Throughput

Fig. 6. Constrained optimization results as a function of end-to-end delay bound in underground environment for volumetric water content.

TABLE II

Overview of Optimal Packet Size Values (Bytes)

\begin{tabular}{|l|l|l|l|}
\hline Environment & $\mathbf{P}_{\text {max }}^{\text {tput }}$ & $\mathbf{P}_{\text {min }}^{\text {eng }}$ & $\mathbf{P}_{\text {min }}^{\text {res }}$ \\
\hline Terrestrial & 152 & 250 & 25 \\
Underwater (Deep) & 668 & 439 & 16 \\
Underwater (Shallow) & 1232 & 1003 & 236 \\
Underground (vwc = 5\%) & 864 & 502 & 23 \\
\hline
\end{tabular}

\section{CONCLUSION}

In this paper, a cross-layer optimization framework for packet size optimization for wireless terrestrial, underwater, and underground sensor networks is developed. Our framework considers medium access collisions, routing decisions, as well as wireless channel affects to determine the optimal packet size. A key result of this analysis is that contrary to conventional wireless networks, longer packets improve the performance of medium access control in WSN. On the other hand, the packet size is limited in terms of energy efficiency due to wireless channel errors. Our cross-layer framework clearly reveals this tradeoff. In this framework, three objective functions are used to investigate the various performance metrics such as throughput, energy consumption per bit, latency, and packet error rate. The results of our analysis show that the routing choices significantly affect overall performance and hence the optimal packet length in WSN due to the multi hop nature of the network. Optimal packet length is found considering this important factor in WSN. Furthermore, the cases for underwater and underground sensor networks are investigated to determine the optimal packet size in these challenged environments. An overview of our results for the three optimization problems; $\mathbf{P}_{\text {max }}^{\text {tput }}, \mathbf{P}_{\text {min }}^{\text {eng }}, \mathbf{P}_{\text {min }}^{\text {res }}$ for terrestrial, underwater, and underground environments are shown in Table II. The developed optimization framework provides a flexible tool to determine the optimal packet size for different application requirements and network topologies.

\section{ACKNOWLEDGEMENT}

This work is supported by the National Science Foundation under contract CNS-0519841 and CCF-0728889.

\section{REFERENCES}

[1] I. F. Akyildiz, W. Su, Y. Sankarasubramaniam, and E. Cayirci, "Wireless Sensor Networks: A Survey", Computer Networks (Elsevier) Journal, Vol. 38, No. 4, pp. 393-422, March 2002.
[2] I. F. Akyildiz, D. Pompili, and T. Melodia, "Underwater Acoustic Sensor Networks: Research Challenges," Ad Hoc Networks Journal (Elsevier), vol. 3, no. 3, pp. 257-279, March 2005.

[3] I. F. Akyildiz, M. C. Vuran, and O. B. Akan, "A Cross-layer Protocol for Wireless Sensor Networks," in Proc. Conference on Information Science and Systems (CISS '06), Princeton, NJ, March 2006.

[4] I. F. Akyildiz and E. Stuntebeck, "Wireless Underground Sensor Networks: Research Challenges," Ad Hoc Networks Journal (Elsevier)," vol. 4, no. 6, pp. 669-686, Nov. 2006.

[5] Y. Barowski, S. Biaz, P. Agrawal, "Towards the Performance Analysis of IEEE 802.11 in Multi-hop Ad-Hoc Networks," in Proc. IEEE Wireless Communications and Networking Conference, vol. 1, pp. 100 - 106, March 2005.

[6] G. Bianchi, "Performance Analysis of the IEEE 802.11 Distributed Coordination Function," IEEE Journal on Selected Areas in Communications, vol. 18 , no. 3 , pp. 535 - 547, March 2000

[7] M. Chitre, J. Potter, and O. Heng, "Underwater Acoustic Channel Characterization for Medium-Range Shallow Water Communications," in Proc. OCEANS '04 MTS/IEEE/TECHNO-OCEAN'04, vol. 1, pp. 4045, Nov. 2004.

[8] J. Choi, J. So, and Y. Ko, "Numerical Analysis of IEEE 802.11 Broadcast Scheme in Multihop Wireless Ad Hoc Networks," in Proc. International Conference on Information Networking, pp. 1-10, 2005.

[9] Crossbow Corp., "Mica2 Datasheet," http://www.xbow.com

[10] S. Haykin, Communication Systems, 3rd ed., Wiley Publishers, 1994.

[11] IEEE 802.15.4, "Wireless Medium Access Control (MAC) and Physical Layer (PHY) Specifications for Low-Rate Wireless Personal Area Networks (LR-WPANs)," October 2003.

[12] P. Lettieri, M. B. Srivastava, "Adaptive frame length control for improving wireless link throughput, range, and energy efficiency," in Proc. IEEE INFOCOM 1998, vol. 2, pp. 564 -571, April 1998.

[13] L. Li, M. C. Vuran, and I. F. Akyildiz, "Characteristics of Underground Channel for Wireless Underground Sensor Networks," in Proc. Med-HocNet '07, Corfu, Greece, June 2007.

[14] F. Poppe, D. De Vleeschauwer, G. H. Petit, "Choosing the UMTS airinterface parameters, the voice packet size and the dejitteringdelay for a voice-over-IP call between a UMTS and a PSTN party,"in Proc. IEEE INFOCOM 2001, vol. 2, pp. 805 -814, April2001.

[15] Y. Sankarasubramaniam, I. F. Akyildiz, S. W. McLaughlin, "Energy efficiency based packet size optimization in wireless sensor networks," in Proc. IEEE Internal Workshop on Sensor Network Protocols and Applications, pp. $1-8,2003$.

[16] K. Schwieger, A. Kumar, G. Fettweis, "On the Impact of the Physical Layer on Energy Consumption in Sensor Networks," in Proc. EWSN '05, pp. 13 - 24, Feb. 2005.

[17] K. Seada, M. Zuniga, A. Helmy, B. Krishnamachari, "Energy-efficient Forwarding Strategies for Geographic Routing in Lossy Wireless Sensor Networks,", in Proc. ACM Sensys '04, November 2004.

[18] E. Shih, et al., "Physical Layer Driven Protocol and Algorithm Design for Energy-efficient Wireless Sensor Networks," in Proc. ACM Mobicom 2001, pp. 272-286, Rome, Italy, July 2001.

[19] M. C. Vuran and I. F. Akyildiz, "Cross Layer Analysis of Error Control in Wireless Sensor Networks,' in Proc. IEEE SECON '06, Reston, VA, September 2006.

[20] M. Zuniga, B. Krishnamachari, "Analyzing the Transitional Region in Low Power Wireless Links," in Proc. IEEE SECON '04, pp. 517 - 526, Oct. 2004 